Imperial/TP/94-95/19

DAMTP 95-16

\title{
Continuous Histories and the History Group in Generalised Quantum Theory
}

\author{
C.J. Isham円 \\ Blackett Laboratory \\ Imperial College \\ South Kensington \\ London SW7 2BZ \\ United Kingdom \\ and \\ N. Linden 2 \\ D.A.M.T.P. \\ University of Cambridge \\ Cambridge CB3 9EW \\ United Kingdom
}

March 1995

\begin{abstract}
We treat continuous histories within the histories approach to generalised quantum mechanics. The essential tool is the 'history group': the analogue, within the generalised history scheme, of the canonical group of single-time quantum mechanics.
\end{abstract}

${ }^{1}$ email: c.isham@ic.ac.uk

${ }^{2}$ email: n.linden@newton.cam.ac.uk 


\section{Introduction}

In this paper we wish to illustrate and develop our algebraic scheme [1, 2, 3] for the consistent-histories approach to quantum theory by extending it to include histories that are continuous (rather than discrete) functions of time. We work within the approach to generalised quantum theory pioneered by Griffiths [4], Omnès [5, 6, 7, 8, 9, 10] and Gell-Mann and Hartle [11, 12, 13, 14, 15, 16, 17] which starts from an observation in conventional quantum theory concerning the joint probability of finding each of a time-ordered sequence of properties $\alpha=\alpha_{t_{1}}, \alpha_{t_{2}}, \ldots, \alpha_{t_{n}}$ with $t_{1}<t_{2}<\cdots<t_{n}$ (we shall call a sequence of this type a homogeneous history, and refer to the sequence of times as the temporal support of the history). Namely, if the initial state at time $t_{0}$ is a density matrix $\rho_{t_{0}}$ then the joint probability of finding all the properties in an appropriate sequence of measurements is

$$
\operatorname{Prob}\left(\alpha_{t_{1}}, \alpha_{t_{2}}, \ldots, \alpha_{t_{n}} ; \rho_{t_{0}}\right)=\operatorname{tr}_{\mathcal{H}}\left(\tilde{C}_{\alpha}^{\dagger} \rho_{t_{0}} \tilde{C}_{\alpha}\right)
$$

where the 'class' operator $\tilde{C}_{\alpha}$ is given in terms of the Schrödinger-picture projection operators $\alpha_{t_{i}}$ as

$$
\tilde{C}_{\alpha}:=U\left(t_{0}, t_{1}\right) \alpha_{t_{1}} U\left(t_{1}, t_{2}\right) \alpha_{t_{2}} \ldots U\left(t_{n-1}, t_{n}\right) \alpha_{t_{n}} U\left(t_{n}, t_{0}\right)
$$

and where $U\left(t, t^{\prime}\right)=e^{-i\left(t-t^{\prime}\right) H / \hbar}$ is the unitary time-evolution operator from time $t$ to $t^{\prime}$. Note that our operator $\tilde{C}_{\alpha}$ is the adjoint of the operator $C_{\alpha}$ used by Gell-Mann and Hartle.

The main assumption of the consistent-histories interpretation of quantum theory is that, under appropriate conditions, the probability assignment (1.1) is still meaningful for a closed system, with no external observers or associated measurement-induced state-vector reductions (thus signalling a move from 'observables' to 'beables'). The satisfaction or otherwise of these conditions (the 'consistency' of a complete set of histories: see below) is determined by the behaviour of the decoherence functional $d_{(H, \rho)}$. This is the complex-valued function of pairs of homogeneous histories $\alpha=\left(\alpha_{t_{1}}, \alpha_{t_{2}}, \ldots, \alpha_{t_{n}}\right)$ and $\beta=\left(\beta_{t_{1}^{\prime}}, \beta_{t_{2}^{\prime}}, \ldots, \beta_{t_{m}^{\prime}}\right)$ defined as

$$
d_{(H, \rho)}(\alpha, \beta)=\operatorname{tr}\left(\tilde{C}_{\alpha}^{\dagger} \rho \tilde{C}_{\beta}\right)
$$

where the temporal supports of $\alpha$ and $\beta$ need not be the same. Note that, as suggested by the notation $d_{(H, \rho)}$, both the initial state and the dynamical 
structure (i.e., the Hamiltonian $H$ ) are coded in the decoherence functional. In our approach, a history of the type $\left(\alpha_{t_{1}}, \alpha_{t_{2}}, \ldots, \alpha_{t_{n}}\right)$ is just a 'passive', time-ordered sequence of propositions. Thinking of the Schrödinger-picture projectors $\alpha_{t_{1}}, \alpha_{t_{2}}, \ldots$ as representing propositions, the homogeneous history $\left(\alpha_{t_{1}}, \alpha_{t_{2}}, \ldots, \alpha_{t_{n}}\right)$ can read as the sequential proposition " $\alpha_{t_{1}}$ is true at time $t_{1}$, and then $\alpha_{t_{2}}$ is true at time $t_{2}$, and then ..., and then $\alpha_{t_{n}}$ is true at time $t_{n} "$.

An important suggestion by Gell-Mann and Hartle is that, in the generalised theory, a history should be regarded as a fundamental entity in its own right, not necessarily just a time-ordered sequence of projection operators. The physical results are obtained by calculating the decoherence functional, now defined as a complex-valued function of pairs of histories that satisfies certain algebraic conditions. When applied to standard quantum theory, a significant technical supposition is that the class of histories should be extended to include 'inhomogeneous' histories, i.e., propositions obtained by applying the logical 'or' operation to homogeneous histories (for example, " $\alpha_{t_{1}}$ is true at time $t_{1}$, and then $\alpha_{t_{2}}$ is true at time $t_{2}$, or, $\beta_{t_{1}^{\prime}}$ is true at time $t_{1}^{\prime}$, and then $\beta_{t_{2}^{\prime}}$ is true at time $t_{2}^{\prime \prime}$.

In previous papers [1, 2, 3] we have argued that the basic ingredients of this generalised quantum theory should be viewed as the set of histories $\mathcal{U P}$ (or, more accurately, the set of propositions about histories) and the set of decoherence functionals $\mathcal{D}$, with the pair $(\mathcal{U P}, \mathcal{D})$ being regarded as the analogue in the history theory of the pair $(\mathcal{L}, \mathcal{S})$ in standard quantum theory, where $\mathcal{L}$ is the lattice of propositions and $\mathcal{S}$ the space of states on $\mathcal{L}$.

As explained in [1, 2], there are cogent reasons for postulating that the natural mathematical structure on the set of histories, $\mathcal{U P}$, is that of an orthoalgebra [18], with the three orthoalgebra operations $\oplus, \neg$ and $<$ corresponding respectively to the disjoint sum, negation and coarse-graining operations invoked by Gell-Mann and Hartle. The properties of the decoherence functional $d: \mathcal{U P} \times \mathcal{U P} \rightarrow \mathbb{C}$ are

1. Hermiticity: $d(\alpha, \beta)=d(\beta, \alpha)^{*}$ for all $\alpha, \beta \in \mathcal{U P}$.

2. Positivity: $d(\alpha, \alpha) \geq 0$ for all $\alpha \in \mathcal{U P}$.

3. Additivity: if $\alpha$ and $\beta$ are disjoint then, for all $\gamma, d(\alpha \oplus \beta, \gamma)=d(\alpha, \gamma)+$ $d(\beta, \gamma)$. If appropriate, this can be extended to countable sums. 


\section{Normalisation: $d(1,1)=1$.}

One important motivation for our framework is the fact that discretetime histories in quantum mechanics can indeed be given the structure of an orthoalgebra. The key idea is that an $n$-time, homogeneous history proposition $\left(\alpha_{t_{1}}, \alpha_{t_{2}}, \ldots, \alpha_{t_{n}}\right)$ can be associated with the operator $\alpha_{t_{1}} \otimes \alpha_{t_{2}} \cdots \otimes \alpha_{t_{n}}$ which is a genuine projection operator on the $n$-fold tensor product $\mathcal{H}_{t_{1}} \otimes$ $\mathcal{H}_{t_{2}} \otimes \cdots \otimes \mathcal{H}_{t_{n}}$ of $n$-copies of the Hilbert-space $\mathcal{H}$ on which the canonical theory is defined [1, 2].

In this paper we investigate how continuous-time history propositions in standard quantum mechanics can be treated within this framework. This raises two immediate issues, both of which we address:

1. the construction of a continuous analogue of the finite product of projection operators used in the definition of the class operator in (1.2);

2. the construction of a continuous analogue of the finite tensor product of projection operators used to associate a homogeneous history with a projection operator.

We shall approach this task with the aid of a tool that we believe is of considerable interest and importance in its own right. This is a history group: an analogue for the history theory of the canonical group used in single-time quantum mechanics. The key idea is that a unitary representation of the appropriate history group leads naturally to an orthoalgebra of projection operators that are to be interpreted as propositions about the 'histories' of that theory. These projection operators are the elements of the spectral representations of the self-adjoint generators of the Lie algebra of the group, or other operators closely related to them. In the case of standard, but continuous-time, quantum theory we seek a history group whose representations yield projection operators that can be associated with propositions about continuous-time histories.

We will demonstrate the existence of such a group for the standard quantum mechanics of a particle moving on the real line, and also construct the decoherence functionals that permit the potential assignment of probabilities to suitably coarse-grained histories for this system. As in the case of discrete-time histories [3], we show that the decoherence functional may be 
written on a tensor product space in terms of an operator $X$ that carries all the information about dynamics. This emphasises the fact that, within the history approach, dynamical evolution is described by a single operator rather than the usual one-parameter family of unitary operators.

\section{Kinematics}

\subsection{The History Group}

As motivation for what follows, let us consider $n$-time quantum mechanics of a particle moving on the line $\mathbb{R}$. As explained in the Introduction, a homogeneous history $\alpha=\left(\alpha_{t_{1}}, \alpha_{t_{2}}, \ldots, \alpha_{t_{n}}\right)$ can be associated with a projection operator $\alpha_{t_{1}} \otimes \alpha_{t_{2}} \cdots \otimes \alpha_{t_{n}}$ on the $n$-fold tensor product $\mathcal{V}_{n}=\mathcal{H}_{t_{1}} \otimes \mathcal{H}_{t_{2}} \cdots \mathcal{H}_{t_{n}}$ of $n$-copies of the Hilbert-space $\mathcal{H}$ of the canonical theory. Although we have not made direct use of it in our work so far, it is clear that, since $\mathcal{H}$ carries a representation of the Heisenberg-Weyl group with Lie algebra (we set $\hbar=1$ )

$$
[x, p]=i,
$$

the Hilbert space $\mathcal{V}_{n}$ carries a unitary representation of the $n$-fold product group with Lie algebra generators satisfy

$$
\begin{aligned}
{\left[x_{k}, x_{m}\right] } & =0 \\
{\left[p_{k}, p_{m}\right] } & =0 \\
{\left[x_{k}, p_{m}\right] } & =i \delta_{k m}
\end{aligned}
$$

with $k, m=1,2, \ldots, n$. Thus, although the vectors of $\mathcal{V}_{n}$ are not directly related to decoherence functionals (which can be viewed as the history analogues of states in single-time quantum theory), it is clear that the Hilbert space $\mathcal{V}_{n}$ carries a representation of the 'history group' with Lie algebra (2.2 2.4). In fact, we could turn the discussion around and define the history version of $n$-time quantum mechanics by starting with (2.2 2.4). In this approach, $\mathcal{V}_{n}$ arises as a representation space for (2.2 2.4), and then any tensor products $\alpha_{t_{1}} \otimes \alpha_{t_{2}} \otimes \cdots \otimes \alpha_{t_{n}}$ corresponding to sequential histories of values of position or momentum (or linear combinations of them) are indeed elements of the spectral representations of this Lie algebra. 
This is the approach that we have found fruitful for discussing continuoustime histories. Specifically, motivated by (2.2 2.4), we start with the historygroup with the following Lie algebra

$$
\begin{aligned}
{\left[x_{t_{1}}, x_{t_{2}}\right] } & =0 \\
{\left[p_{t_{1}}, p_{t_{2}}\right] } & =0 \\
{\left[x_{t_{1}}, p_{t_{2}}\right] } & =i \delta\left(t_{1}-t_{2}\right)
\end{aligned}
$$

where $-\infty \leq t_{1}, t_{2} \leq \infty$. The operators appearing here should not be confused with the family $x(t), p(t)$ of Heisenberg-picture operators in normal quantum theory. Indeed, it should be noted that, although we are studying the history version of quantum mechanics on $\mathbb{R}$, we are led to a Liealgebra which usually arises as the canonical commutation relations of a one-dimensional quantum field theory! In what follows, we shall need to be a little more careful about how this algebra is specified; in particular, we introduce a test-function space which we take to be the space $L_{\mathbb{R}}^{2}(\mathbb{R})$ of real, square-integrable, functions on $\mathbb{R}$. Hence (2.5 2.7) are replaced by

$$
\begin{aligned}
& {\left[x_{f}, x_{g}\right]=0} \\
& {\left[p_{f}, p_{g}\right]=0} \\
& {\left[x_{f}, p_{g}\right]=i(f, g)}
\end{aligned}
$$

where $f, g \in L_{\mathbb{R}}^{2}(\mathbb{R})$ and $(f, g) \stackrel{\text { def }}{=} \int_{-\infty}^{\infty} f(x) g(x) d x$.

\subsection{The Hilbert Space $\mathcal{V}_{\text {cts }}$}

In order to understand how the representations of (2.5 2.7) lead to an appropriate notion of a continuous tensor product of copies of the Hilbert space $L^{2}(\mathbb{R})$ of canonical quantum mechanics, we need to introduce the concept of an exponential Hilbert space 19, 20, 21, 22, 23. Given a Hilbert space $\mathcal{K}$, the exponential Hilbert space $e^{\mathcal{K}}$ is constructed as follows. Let $(\otimes \mathcal{K})^{n}$ denote the $n$-fold tensor product of $\mathcal{K}$ with itself, and let $(\otimes \mathcal{K})_{S}^{n}$ be the subspace of $(\otimes \mathcal{K})^{n}$ spanned by the vectors $(\otimes \phi)^{n}, \phi \in \mathcal{K}$, where $(\otimes \mathcal{K})^{0}=(\otimes \mathcal{K})_{S}^{0}$ is defined to be the one-dimensional Hilbert space of the complex numbers. Then the exponential Hilbert space is defined as $e^{\mathcal{K}} \stackrel{\text { def }}{=} \oplus_{n=0}^{\infty}(\otimes K)_{S}^{n}$. Of course, the space $e^{\mathcal{K}}$ is also known as the 'bosonic Fock space' over $\mathcal{K}$. It is more 
usual to define $(\otimes \mathcal{K})_{S}^{n}$ as the subspace of $(\otimes \mathcal{K})^{n}$ spanned by symmetrised product vectors; however, the definition used here is equivalent (for example, in $(\otimes \mathcal{K})^{2}$, one may obtain $a \otimes b+b \otimes a$ as $(a+b) \otimes(a+b)-a \otimes a-b \otimes b$. $)$

Let $|\exp \phi\rangle$ denote the (non-normalised) coherent state vector

$$
\oplus_{n=0}^{\infty}(n !)^{-\frac{1}{2}}(\otimes|\phi\rangle)^{n}
$$

Then the inner product in $e^{\mathcal{K}}$ is given by

$$
\langle\exp \phi \mid \exp \psi\rangle_{e^{\mathcal{K}}}=e^{\langle\phi, \psi\rangle_{\mathcal{K}}} \text {. }
$$

As shown in [19], the vectors $|\exp \phi\rangle$ are total in $e^{\mathcal{K}}$ : a fact we shall use frequently later.

Let $\mathcal{T}$ denote the complexification of the real test function space used to define smeared canonical commutation relations, so that we can write

$$
\begin{aligned}
{\left[a_{f}, a_{g}\right] } & =0 \\
{\left[a_{f}^{\dagger}, a_{g}^{\dagger}\right] } & =0 \\
{\left[a_{f}, a_{g}^{\dagger}\right] } & =\langle f, g\rangle_{\mathcal{T}} .
\end{aligned}
$$

where $\langle f, g\rangle$ denotes the associated scalar product. Then the Fock representation of these creation and annihilation operators is defined on the exponential Hilbert space $e^{\mathcal{T}}$ so that, for example, the matrix element of $a_{f}$ is

$$
\left\langle\exp \phi\left|a_{f}\right| \exp \psi\right\rangle_{e^{\mathcal{T}}}=\langle f, \psi\rangle e^{\langle\phi, \psi\rangle_{\mathcal{T}}} .
$$

A special case is when $\mathcal{T}$ is $\mathbb{C}$, which we shall treat explicitly here as the results are needed later. The usual normalised coherent states are

$$
|z\rangle \stackrel{\text { def }}{=} e^{-\frac{1}{2}|z|^{2}+z a^{\dagger}}|0\rangle,
$$

and satisfy $\langle z \mid w\rangle=e^{-\frac{1}{2}|z|^{2}-\frac{1}{2}|w|^{2}+z^{*} w}$. These normalised states are related to the exponential vectors by $|\exp z\rangle=e^{\frac{1}{2}|z|^{2}}|z\rangle$, and the Hilbert space exp $\mathbb{C}$ is isomorphic to $L^{2}(\mathbb{R})$ via

$$
\begin{aligned}
\exp \mathbb{C} & \simeq L^{2}(\mathbb{R}, d x) \\
|\exp z\rangle & \mapsto\langle x \mid \exp z\rangle=(2 \pi)^{-\frac{1}{4}} e^{z x-\frac{1}{2} z^{2}-\frac{1}{4} x^{2}}
\end{aligned}
$$


Our reason for introducing exponential Hilbert spaces is the existence of a particularly convenient construction of a continuous tensor product of a one-parameter family of them. In general, given a family $t \mapsto \mathcal{H}_{t}$ of Hilbert spaces, we try to define an inner product, following Streater, 21] as

$$
\left\langle\otimes_{t} u_{t} \mid \otimes_{t} v_{t}\right\rangle_{\otimes_{t} \mathcal{H}_{t}} \stackrel{\text { def }}{=} e^{\int_{-\infty}^{\infty} \log \left\langle u_{t}, v_{t}\right\rangle_{\mathcal{H}_{t}} d t}
$$

if this expression makes sense. This is intended to be the continuous analogue of the inner product between discrete tensor products of vectors

$$
\left\langle u_{1} \otimes u_{2} \otimes \cdots \otimes u_{n} \mid v_{1} \otimes v_{2} \otimes \cdots \otimes v_{n}\right\rangle \stackrel{\text { def }}{=} \prod_{i=1}^{n}\left\langle u_{i}, v_{i}\right\rangle \equiv e^{\sum_{i=1}^{n} \log \left\langle u_{i}, v_{i}\right\rangle} .
$$

If $\mathcal{H}_{t}$ is an exponential Hilbert space $\mathcal{H}_{t}=e^{\mathcal{K}_{t}}$, then the construction works since

$$
\left\langle\exp \phi_{t} \mid \exp \psi_{t}\right\rangle_{e^{\mathcal{K}_{t}}}=e^{\left\langle\phi_{t}, \psi_{t}\right\rangle_{\mathcal{K}_{t}}}
$$

and so the definition of the scalar product on the continuous tensor product of copies of $e^{\mathcal{K}_{t}}$ as

$$
\left\langle\otimes_{t} \exp \phi_{t} \mid \otimes_{t} \exp \psi_{t}\right\rangle_{\otimes_{t} e^{\mathcal{K}_{t}}} \stackrel{\text { def }}{=} e^{\int_{-\infty}^{\infty}\left\langle\phi_{t}, \psi_{t}\right\rangle_{\mathcal{K}_{t}} d t}
$$

is well-defined.

Furthermore, the scalar product $\int_{-\infty}^{\infty}\left\langle\phi_{t}, \psi_{t}\right\rangle_{\mathcal{K}_{t}} d t$ is the inner product on the direct-integral Hilbert space $\int^{\oplus} \mathcal{K}_{t}$, and hence

$$
\left\langle\otimes_{t} \exp \phi_{t} \mid \otimes_{t} \exp \psi_{t}\right\rangle_{\otimes_{t} e^{\mathcal{K}_{t}}}=\langle\exp \phi(\cdot) \mid \exp \psi(\cdot)\rangle_{\exp \int}{ }^{\oplus} \mathcal{K}_{t}
$$

In fact, there is an isomorphism

$$
\begin{aligned}
\otimes_{t} \exp \mathcal{K}_{t} & \simeq \exp \int^{\oplus} \mathcal{K}_{t} \\
\otimes_{t}\left|\exp \phi_{t}\right\rangle & \mapsto|\exp \phi(\cdot)\rangle
\end{aligned}
$$

Let us use these ideas now to see how the Fock representation of (2.8 2.10) with the test-function space $L_{\mathbb{R}}^{2}(\mathbb{R})$ leads to a continuous tensor product space $\otimes_{t} L_{t}^{2}(\mathbb{R})$. The complexification of the real vector space $L_{\mathbb{R}}^{2}(\mathbb{R})$ is just 
$L^{2}(\mathbb{R})$, and hence the Fock representation of $(2.132 .15)$ is on the space $\exp \left(L^{2}(\mathbb{R})\right)$. However, $L^{2}(\mathbb{R})$ is isomorphic to the direct integral $\int{ }^{\oplus} \mathbb{C}_{t} d t$ via

$$
\begin{aligned}
\int^{\oplus} \mathbb{C}_{t} d t & \simeq L^{2}(\mathbb{R}, d t) \\
\int^{\oplus} w_{t} d t & \mapsto w(\cdot)
\end{aligned}
$$

so that we may think of the Hilbert space as

$$
\exp \left(L^{2}(\mathbb{R})\right) \simeq \exp \int^{\oplus} \mathbb{C}_{t} d t .
$$

On the other hand, (2.24) means that

$$
\exp \int^{\oplus} \mathbb{C}_{t} d t \simeq \otimes_{t}\left(\exp \mathbb{C}_{t}\right),
$$

and (2.18) shows that

$$
\exp \mathbb{C}_{t} \simeq L_{t}^{2}(\mathbb{R}) .
$$

Hence we see that the Hilbert space (i.e., Fock space) upon which the history algebra (2.8 2.10) is naturally represented, is isomorphic to $\otimes_{t} L_{t}^{2}(\mathbb{R})$, and therefore the space $\mathcal{V}_{\text {cts }}$ which carries the propositions/projection operators of our continuous history theory is

$$
\mathcal{V}_{\text {cts }} \stackrel{\text { def }}{=} \otimes_{t}\left(L_{t}^{2}(\mathbb{R})\right) \simeq \exp \left(L^{2}(\mathbb{R}, d t)\right) .
$$

\subsection{History Propositions}

Having found the Hilbert space $\mathcal{V}_{\text {cts }}$, the next step is to identify some projection operators on $\mathcal{V}_{\text {cts }}$ that have a clear physical meaning. In standard canonical quantum theory, the Lie algebra of the canonical group provides a preferred class of classical observables that are to be quantised as selfadjoint operators. A typical proposition is of the form that the value of such an observable lies in some specified range. This proposition is represented mathematically by the appropriate projector in the spectral representation of the associated self-adjoint operator.

It would be possible to develop the analogue of this idea in the history theory. In particular, using the smeared form (2.8 2.10) of the history algebra, it is natural to consider the quantum representation of propositions of 
the form " $x_{f}+p_{g}$ lies in a subset $\Delta \subset \mathbb{R}$ ", where $f$ and $g$ are test functions. Propositions of this type clearly deal with the time averages of position and momentum.

However, in this paper we shall adopt a somewhat different approach, based on the observation that coherent states play an intimate role in the construction of the continuous tensor product. This suggests that it may be productive to focus on projectors onto such states. Thus the task is to define a continuous tensor product $\otimes_{t} P_{\lambda(t)}$ where $t \mapsto \lambda(t)$ is a complex-valued map of bounded variation from $\mathbb{R}$, and where, for each $t \in \mathbb{R}$, the operator $P_{\lambda(t)} \stackrel{\text { def }}{=}|\lambda(t)\rangle\langle\lambda(t)|$ is the projector onto the normalised coherent state $|\lambda(t)\rangle$.

The first obvious thing to try is

$$
\left(\otimes_{t} P_{\lambda(t)}\right)\left(\otimes_{t}|\exp \mu(t)\rangle\right) \stackrel{\text { def }}{=} \otimes_{t}\left(P_{\lambda(t)}|\exp \mu(t)\rangle\right)
$$

where we recall that the exponential states $|\exp \mu(t)\rangle \in L_{t}^{2}(\mathbb{R})$ are given in terms of the normalised coherent states $|\mu(t)\rangle$ as

$$
|\exp \mu(t)\rangle=e^{\frac{1}{2}|\mu|^{2}}|\mu(t)\rangle
$$

and with

$$
\langle\lambda(t) \mid \mu(t)\rangle=e^{\lambda(t)^{*} \mu(t)-\frac{1}{2}|\lambda(t)|^{2}-\frac{1}{2}|\mu(t)|^{2}} .
$$

Thus

$$
\begin{aligned}
P_{\lambda(t)}|\exp \mu(t)\rangle & =e^{-\frac{1}{2}|\lambda(t)|^{2}+\lambda(t)^{*} \mu(t)}|\lambda(t)\rangle \\
& =e^{-\lambda(t)^{*}(\lambda(t)-\mu(t))}|\exp \lambda(t)\rangle
\end{aligned}
$$

and hence

$$
\otimes_{t} P_{\lambda(t)}\left(\otimes_{t}|\exp \mu(t)\rangle\right)=\otimes_{t}\left(e^{-\lambda(t)^{*}(\lambda(t)-\mu(t))}|\exp \lambda(t)\rangle\right) .
$$

However, if $\mu(t)$ and $\lambda(t)$ differ from each other on an open subset of $t$ values, then the continuous product

$$
\prod_{t} e^{-\lambda(t)^{*}(\lambda(t)-\mu(t))}
$$

will not converge. Hence the right-hand side of (2.36) is not defined, and so we cannot use (2.31). 
The difficulty we have found arises from the fact that it is not the continuous product of normalised states $\otimes_{t}|\lambda(t)\rangle$ that is well-defined but rather the continuous product of non-normalised states $\otimes_{t}|\exp \lambda(t)\rangle=\otimes_{t} e^{\frac{1}{2}|\lambda(t)|^{2}}|\lambda(t)\rangle$. This suggests that we identify $\otimes_{t} P_{\lambda(t)}$ with $P_{\otimes_{t}|\exp \lambda(t)\rangle}$, i.e., the projector onto the vector $\otimes_{t}|\exp \lambda(t)\rangle$ in $\otimes_{t} L_{t}^{2}(\mathbb{R})$. Under the identification of $\otimes_{t} L_{t}^{2}(\mathbb{R})$ with $\exp L^{2}(\mathbb{R}, d t)$, this projector is identified with $P_{|\exp \lambda(\cdot)\rangle}=$ $e^{-\langle\lambda, \lambda\rangle}|\exp \lambda(\cdot)\rangle\langle\exp \lambda(\cdot)|$. The action of this projector is

$$
P_{|\exp \lambda(\cdot)\rangle}|\exp \mu(\cdot)\rangle=e^{\langle\lambda, \mu-\lambda\rangle}|\exp \lambda(\cdot)\rangle
$$

where

$$
\langle\lambda, \mu-\lambda\rangle=\int_{-\infty}^{\infty} \lambda^{*}(t)(\mu(t)-\lambda(t)) d t .
$$

This operator is well-defined, and may easily be seen to satisfy

$$
P_{|\exp \lambda(\cdot)\rangle} P_{|\exp \lambda(\cdot)\rangle}=P_{|\exp \lambda(\cdot)\rangle} \quad \text { and } \quad P_{|\exp \lambda(\cdot)\rangle}^{\dagger}=P_{|\exp \lambda(\cdot)\rangle},
$$

as it should.

In addition to the projectors $P_{|\exp \lambda(\cdot)\rangle}$, we might wish to handle propositions about histories that involve only a finite time interval $[a, b]$. Of course, one possibility is to start with the Hilbert space $L^{2}[a, b]$ rather than $L^{2}(\mathbb{R})$. However, what we want is a single history theory that can accommodate all possible finite time intervals, not just one.

Since the continuous product of copies of the single projector $P_{|\lambda(a)\rangle}$ is just itself, one might be tempted to define $P_{|\exp \lambda(t)\rangle}$ for all $t$ as above but then impose the constraints

$$
\begin{aligned}
\lambda(t) & =\lambda(a) \quad t<a, \\
& =\lambda(b) \quad t>b .
\end{aligned}
$$

However, the fact that the function $\lambda$ is supposed to be a member of $L^{2}(\mathbb{R}, d t)$ leads to a difficulty: unless $\lambda(a)=\lambda(b)=0$, the function $\lambda$ defined above will not be square-integrable, and so the class of projectors $\left\{P_{|\exp \lambda(\cdot)\rangle}\right\}$ does not contain operators of this type.

What we would really like to do is to construct projectors that are equal to $P_{|\exp \lambda(\cdot)\rangle}$ in the 'active' region $[a, b]$, and are equal to the unit operator outside $[a, b]$. This leads us to define the following operator $P_{|\exp \lambda(\cdot)\rangle}^{[a, b]}$ :

$$
P_{|\exp \lambda(\cdot)\rangle}^{[a, b]}|\exp \mu(\cdot)\rangle \stackrel{\text { def }}{=} \exp \left(\int_{a}^{b} \lambda^{*}(t)(\mu(t)-\lambda(t)) d t\right)|\exp \lambda \star \mu(\cdot)\rangle
$$


where

$$
(\lambda \star \mu)(t) \stackrel{\text { def }}{=} \begin{cases}\lambda(t) & \text { if } t \in[a, b] \\ \mu(t) & \text { otherwise. }\end{cases}
$$

A little work is required to show that $P_{|\exp \lambda(\cdot)\rangle}^{[a, b]}$ is a genuine projection operator on $\mathcal{V}_{\text {cts }}=\exp \left(L^{2}(\mathbb{R})\right)$. Firstly consider

$$
\begin{aligned}
P_{|\exp \lambda(\cdot)\rangle}^{[a, b]} P_{|\exp \lambda(\cdot)\rangle}^{[a, b]}|\exp \mu(\cdot)\rangle= & e^{\int_{a}^{b} \lambda^{*}(t)(\mu(t)-\lambda(t)) d t} P_{|\exp \lambda(\cdot)\rangle}^{[a, b]}|\exp \lambda \star \mu(\cdot)\rangle \\
= & e^{\int_{a}^{b} \lambda^{*}(t)(\mu(t)-\lambda(t)) d t} e^{\int_{a}^{b} \lambda^{*}(t)((\lambda \star \mu)(t)-\lambda(t)) d t} \\
& \times|\exp \lambda \star(\lambda \star \mu)(\cdot)\rangle .
\end{aligned}
$$

Now, if $t \in[a, b]$, then $\lambda \star \mu(t)=\lambda(t)$, and hence the second exponent vanishes. Furthermore, if $t \in[a, b]$, then $\lambda \star(\lambda \star \mu)(t)=\lambda(t)$, and if $t \notin[a, b]$, then $\lambda \star(\lambda \star \mu)(t)=\mu(t)$; hence $\lambda \star(\lambda \star \mu)=(\lambda \star \mu)$. Thus we find

$$
\begin{aligned}
P_{|\exp \lambda(\cdot)\rangle}^{[a, b]} P_{|\exp \lambda(\cdot)\rangle}^{[a, b]}|\exp \mu(\cdot)\rangle & =e^{\int_{a}^{b} \lambda^{*}(t)(\mu(t)-\lambda(t)) d t}|\exp (\lambda \star \mu)(\cdot)\rangle \\
& =P_{|\exp \lambda(\cdot)\rangle}^{[a, b]}|\exp \mu(\cdot)\rangle .
\end{aligned}
$$

Since this is true for all $|\exp \mu(\cdot)\rangle$ (i.e., for all elements of a total set of vectors) this implies that

$$
P_{|\exp \lambda(\cdot)\rangle}^{[a, b]} P_{|\exp \lambda(\cdot)\rangle}^{[a, b]}=P_{|\exp \lambda(\cdot)\rangle}^{[a, b]} .
$$

The other property we need to prove is self-adjointness. To this end, consider the matrix elements

$$
\begin{aligned}
& \left\langle\exp \mu(\cdot)\left|P_{|\exp \lambda(\cdot)\rangle}^{[a, b]}\right| \exp \nu(\cdot)\right\rangle=e^{\int_{a}^{b} \lambda^{*}(t)(\nu(t)-\lambda(t)) d t}\langle\exp \mu(\cdot) \mid \exp \lambda \star \nu(\cdot)\rangle \\
& =e^{\int_{a}^{b} \lambda^{*}(t)(\nu(t)-\lambda(t)) d t} \\
& \times \quad e^{\int_{-\infty}^{\infty} \mu^{*}(t) \lambda \star \nu(t) d t} .
\end{aligned}
$$

Now

$$
\int_{-\infty}^{\infty} \mu^{*}(t) \lambda \star \nu(t) d t=\int_{-\infty}^{\infty} \mu^{*}(t) \nu(t) d t+\int_{a}^{b} \mu^{*}(t)(\lambda(t)-\nu(t)) d t
$$

and so

$$
\left\langle\exp \mu(\cdot)\left|P_{|\exp \lambda(\cdot)\rangle}^{[a, b]}\right| \exp \nu(\cdot)\right\rangle=e^{\int_{a}^{b}\left(\mu^{*}(t)-\lambda^{*}(t)\right)(\lambda(t)-\nu(t)) d t} e^{\int_{-\infty}^{\infty} \mu^{*}(t) \nu(t) d t} .
$$


Now consider

$$
\begin{aligned}
\left\langle\exp \mu(\cdot)\left|\left(P_{|\exp \lambda(\cdot)\rangle}^{[a, b]}\right)^{\dagger}\right| \exp \nu(\cdot)\right\rangle= & \left\langle P_{|\exp \lambda(\cdot)\rangle}^{[a, b]} \exp \mu(\cdot) \mid \exp \nu(\cdot)\right\rangle \\
= & e^{\int_{a}^{b} \lambda(t)(\mu(t)-\lambda(t))^{*} d t} \\
& \times\langle\exp \lambda \star \mu(\cdot) \mid \exp \nu(\cdot)\rangle \\
= & e^{\int_{a}^{b} \lambda(t)(\mu(t)-\lambda(t))^{*} d t} \\
& \times e^{\int_{-\infty}^{\infty}(\lambda \star \mu(t))^{*} \nu(t) d t} .
\end{aligned}
$$

But

$$
\int_{-\infty}^{\infty}(\lambda \star \mu(t))^{*} \nu(t) d t=\int_{-\infty}^{\infty} \mu^{*}(t) \nu(t) d t+\int_{a}^{b}(\lambda(t)-\mu(t))^{*} \nu(t) d t
$$

and so

$$
\left\langle\exp \mu(\cdot)\left|\left(P_{|\exp \lambda(\cdot)\rangle}^{[a, b]}\right)^{\dagger}\right| \exp \nu(\cdot)\right\rangle=e^{\int_{a}^{b}\left(\mu^{*}(t)-\lambda^{*}(t)\right)(\lambda(t)-\nu(t)) d t} e^{\int_{-\infty}^{\infty} \mu^{*}(t) \nu(t) d t}
$$

which is equal to 2.49$)$. Thus the (bounded) operator $P_{|\exp \lambda(\cdot)\rangle}^{[a, b]}$ satisfies

$$
P_{|\exp \lambda(\cdot)\rangle}^{[a, b]}=\left(P_{|\exp \lambda(\cdot)\rangle}^{[a, b]}\right)^{\dagger}
$$

and

$$
P_{|\exp \lambda(\cdot)\rangle}^{[a, b]} P_{|\exp \lambda(\cdot)\rangle}^{[a, b]}=P_{|\exp \lambda(\cdot)\rangle}^{[a, b]}
$$

and is hence a projection operator on $\mathcal{V}_{\text {cts }}=\exp \left(L^{2}(\mathbb{R})\right)$.

\section{Dynamics}

\subsection{The decoherence functional in terms of continuous products}

In our formulation of the Gell-Mann and Hartle generalised quantum theory, it is the decoherence functional $d$ that contains all the information about the dynamics. In [3] it was shown that if $\left(\alpha_{t_{1}}, \alpha_{t_{2}}, \ldots, \alpha_{t_{n}}\right)$ and $\left(\beta_{t_{1}^{\prime}}, \beta_{t_{2}^{\prime}}, \ldots, \beta_{t_{m}^{\prime}}\right)$ are discrete-time histories in a standard quantum theory (whose underlying 
Hilbert space is denoted $\mathcal{H})$, then there exists an operator $X$ on $\left(\otimes^{n} \mathcal{H}\right) \otimes$ $\left(\otimes^{m} \mathcal{H}\right)$ such that the decoherence functional may be written as

$$
d(\alpha, \beta)=\operatorname{tr}_{\left(\otimes^{n} \mathcal{H}\right) \otimes\left(\otimes^{m} \mathcal{H}\right)}(\alpha \otimes \beta X)
$$

where $X$ is independent of $\alpha \stackrel{\text { def }}{=} \alpha_{t_{1}} \otimes \alpha_{t_{2}} \otimes \cdots \otimes \alpha_{t_{n}}$ and $\beta \stackrel{\text { def }}{=} \beta_{t_{1}^{\prime}} \otimes \beta_{t_{2}^{\prime}} \otimes \cdots \otimes \beta_{t_{m}^{\prime}}$. It was further shown that the above construction could be extended to the full infinite tensor product $\mathcal{V} \Omega \stackrel{\text { def }}{=} \otimes_{t \in \mathbb{R}}^{\Omega} \mathcal{H}_{t}$, the space upon which discrete-time propositions are projection operators. Thus the result $d(\alpha, \beta)=\operatorname{tr}(\alpha \otimes \beta X)$ extends to the set $\mathcal{U P}$ of all discrete-time history propositions in standard quantum theory.

In the same paper we classified the decoherence functionals in the case when $\mathcal{U P}$ is the lattice of propositions $\mathcal{P}(\mathcal{V})$ where the Hilbert space $\mathcal{V}$ has a finite dimension. In particular, we proved that the four axioms of section 1 , namely hermiticity, positivity, additivity and normalisation, suffice to show that every decoherence functional $d$ can be written in the form

$$
d(\alpha, \beta)=\operatorname{tr}_{\mathcal{V} \otimes \mathcal{V}}(\alpha \otimes \beta X)
$$

for some operator $X$ on the tensor product space $\mathcal{V} \otimes \mathcal{V}$.

The purpose of this section is to show that the decoherence functional of standard quantum mechanics with continuous time projections can also be written in the form

$$
d(\mu, \nu)=\operatorname{tr}_{\mathcal{V}_{\mathrm{cts}} \otimes \mathcal{V}_{\mathrm{cts}}}\left(P_{\mu} \otimes P_{\nu} X\right)
$$

where $P_{\mu}$ and $P_{\nu}$ are suitable projection operators on $\mathcal{V}_{\text {cts }}$, and $X$ is an operator on $\mathcal{V}_{\text {cts }} \otimes \mathcal{V}_{\text {cts }}$.

However, before embarking on this discussion we need first to specify what we mean by a continuous product of projectors in standard quantum theory, and then calculate $d(\mu, \nu)$ for these projectors. The previous discussion leads one to think that it might be particularly appropriate to consider continuous products of projectors onto coherent states. Therefore, let $\lambda[a, b] \rightarrow \mathbb{C}$ be a curve of bounded variation, and let us try to define the continuous product of coherent-state projection operators in the Heisenberg picture.

We begin with the simple case where the Hamiltonian is zero, so that there is no difference between Schrödinger picture and Heisenberg picture. 
Hence we are interested in

$$
\tilde{C}_{[a, b]}[\lambda] \stackrel{\text { def }}{=} \prod_{t \in[a, b]} P_{\lambda(t)}
$$

where

$$
P_{\lambda(t)} \stackrel{\text { def }}{=}|\lambda(t)\rangle\langle\lambda(t)|
$$

and $|z\rangle$ denotes the usual normalised coherent state.

In order to give meaning to the right hand side of (3.4) let us start by subdividing the interval $[a, b]$ as $a=t_{1}<t_{2}<\ldots<t_{n}=b$. A finite approximation to $\tilde{C}_{[a, b]}[\lambda]$ is then

$$
\begin{aligned}
P_{\lambda\left(t_{1}\right)} P_{\lambda\left(t_{2}\right)} & \ldots P_{\lambda\left(t_{n}\right)} \\
= & \left|\lambda\left(t_{1}\right)\right\rangle\left\langle\lambda\left(t_{1}\right) \mid \lambda\left(t_{2}\right)\right\rangle\left\langle\lambda\left(t_{2}\right) \mid \lambda\left(t_{3}\right)\right\rangle\left\langle\lambda\left(t_{3}\right)|\ldots| \lambda\left(t_{n}\right)\right\rangle\left\langle\lambda\left(t_{n}\right)\right| \\
= & e^{-\frac{1}{2}\left(\left|\lambda\left(t_{1}\right)\right|^{2}+\left|\lambda\left(t_{n}\right)\right|^{2}\right)-\left(\left|\lambda\left(t_{2}\right)\right|^{2}+\left|\lambda\left(t_{3}\right)\right|^{2}+\ldots\left|\lambda\left(t_{n-1}\right)\right|^{2}\right)} \\
& \quad \times e^{\lambda\left(t_{1}\right)^{*} \lambda\left(t_{2}\right)+\lambda\left(t_{2}\right)^{*} \lambda\left(t_{3}\right) \ldots+\lambda\left(t_{n-1}\right)^{*} \lambda\left(t_{n}\right)} \times|\lambda(a)\rangle\langle\lambda(b)| \\
= & e^{\lambda\left(t_{1}\right)^{*}\left(\lambda\left(t_{2}\right)-\lambda\left(t_{1}\right)\right)+\lambda\left(t_{2}\right)^{*}\left(\lambda\left(t_{3}\right)-\lambda\left(t_{2}\right)\right)+\ldots+\lambda\left(t_{n-1}\right)^{*}\left(\lambda\left(t_{n}\right)-\lambda\left(t_{n-1}\right)\right)} \\
& \times e^{\frac{1}{2}\left(\left|\lambda\left(t_{1}\right)\right|^{2}-\left|\lambda\left(t_{n}\right)\right|^{2}\right)} \times|\lambda(a)\rangle\langle\lambda(b)| .
\end{aligned}
$$

As the subdivision gets finer and finer, the first exponent on the right hand side converges to the Stieltjes integral $\int_{a}^{b} \lambda^{*} d \lambda$, which motivates defining the continuous product (3.4) as

$$
\begin{gathered}
\tilde{C}_{[a, b]}[\lambda] \stackrel{\text { def }}{=} e^{\int_{a}^{b} \lambda^{*} d \lambda} e^{\frac{1}{2}\left(|\lambda(a)|^{2}-|\lambda(b)|^{2}\right)} \times|\lambda(a)\rangle\langle\lambda(b)| \\
=e^{\frac{1}{2} \int_{a}^{b}\left(\lambda^{*} d \lambda-\lambda d \lambda^{*}\right)} \times|\lambda(a)\rangle\langle\lambda(b)| .
\end{gathered}
$$

Note that the operator thus defined satisfies

$$
\tilde{C}_{[a, b]}[\lambda] \tilde{C}_{[b, c]}[\lambda]=\tilde{C}_{[a, c]}[\lambda]
$$

where $a<b<c$. This semigroup property is a natural consistency condition to impose on any definition of a continuous product of projection operators.

We turn now to the case in which the Hamiltonian is non-zero, and first define the Heisenberg-picture operator, with fiducial time $t_{0}$,

$$
P_{\lambda(t)}(t) \stackrel{\text { def }}{=} U\left(t_{0}, t\right) P_{\lambda(t)} U\left(t, t_{0}\right),
$$


so that a finite-time approximation to the product $\prod_{t \in[a, b]} P_{\lambda(t)}(t)$ is

$$
\begin{aligned}
& U\left(t_{0}, t_{1}\right) P_{\lambda\left(t_{1}\right)} U\left(t_{1}, t_{2}\right) P_{\lambda\left(t_{2}\right)} U\left(t_{2}, t_{3}\right) P_{\lambda\left(t_{3}\right)} \ldots P_{\lambda\left(t_{n}\right)} U\left(t_{n}, t_{0}\right) \\
= & U\left(t_{0}, t_{1}\right)\left|\lambda\left(t_{1}\right)\right\rangle\left\langle\lambda\left(t_{1}\right)\left|U\left(t_{1}, t_{2}\right)\right| \lambda\left(t_{2}\right)\right\rangle\left\langle\lambda\left(t_{2}\right)\left|U\left(t_{2}, t_{3}\right)\right| \lambda\left(t_{3}\right)\right\rangle\left\langle\lambda\left(t_{3}\right)\right| \ldots \\
& \left\langle\lambda\left(t_{n-1}\right)\left|U\left(t_{n-1}, t_{n}\right)\right| \lambda\left(t_{n}\right)\right\rangle\left\langle\lambda\left(t_{n}\right)\right| U\left(t_{n}, t_{0}\right) .
\end{aligned}
$$

We now use a standard trick [24] to evaluate the matrix elements for small $\left(t_{r}-t_{r+1}\right)$ :

$$
\begin{aligned}
\left\langle\lambda\left(t_{r}\right)\left|U\left(t_{r}, t_{r+1}\right)\right| \lambda\left(t_{r+1}\right)\right\rangle=\left\langle\lambda\left(t_{r}\right)\left|e^{-i\left(t_{r}-t_{r+1}\right) H / \hbar}\right| \lambda\left(t_{r+1}\right)\right\rangle & \\
\approx & \left\langle\lambda\left(t_{r}\right)\left|1-i\left(t_{r}-t_{r+1}\right) H / \hbar\right| \lambda\left(t_{r+1}\right)\right\rangle \\
\approx & \left\langle\lambda\left(t_{r}\right) \mid \lambda\left(t_{r+1}\right)\right\rangle \times \\
& \left(1-i\left(t_{r}-t_{r+1}\right) H\left(\lambda\left(t_{r}\right), \lambda\left(t_{r+1}\right)\right) / \hbar\right) \\
\approx & \left\langle\lambda\left(t_{r}\right) \mid \lambda\left(t_{r+1}\right)\right\rangle e^{-i\left(t_{r}-t_{r+1}\right) H\left(\lambda\left(t_{r}\right), \lambda\left(t_{r+1}\right)\right) / \hbar}
\end{aligned}
$$

where

$$
H\left(z, z^{\prime}\right) \stackrel{\text { def }}{=} \frac{\left\langle z|H| z^{\prime}\right\rangle}{\left\langle z \mid z^{\prime}\right\rangle},
$$

and where we are not attempting to be rigorous about domains of unbounded operators and the like. Thus, in the limit of finer and finer subdivisions, we again get an expression for the continuous product of projectors as an integral

$$
\begin{aligned}
\tilde{C}_{[a, b]}[\lambda]= & \prod_{t \in[a, b]} P_{\lambda(t)}(t) \\
= & e^{\frac{1}{2}\left(|\lambda(a)|^{2}-|\lambda(b)|^{2}\right)} e^{\int_{a}^{b} \lambda^{*} d \lambda+i / \hbar \int_{a}^{b} H(\lambda(t)) d t} \\
& \quad \times U\left(t_{0}, a\right)|\lambda(a)\rangle\langle\lambda(b)| U\left(b, t_{0}\right)
\end{aligned}
$$

where $H(z) \stackrel{\text { def }}{=} H(z, z)=\langle z|H| z\rangle$. Note that this definition also satisfies the semigroup property (3.8).

We can now use these results to compute the decoherence functional for the histories associated with the paths $t \mapsto \mu(t)$ and $t \mapsto \lambda(t)$, where from now on we will take $a=-\infty$ and $b=\infty$ for simplicity. Thus we define

$$
d(\mu, \nu) \stackrel{\text { def }}{=} \operatorname{tr}\left\{\prod_{t \in \mathbb{R}}\left(P_{\mu(t)}(t)\right)^{\dagger} \rho_{-\infty} \prod_{t \in \mathbb{R}}\left(P_{\nu(t)}(t)\right)\right\}
$$


where $\rho_{-\infty}$ is the initial density matrix. Using our previous results we can calculate this decoherence functional as

$$
d(\mu, \nu)=e^{\int_{-\infty}^{\infty}\left(\nu^{*} d \nu-\mu^{*} d \mu\right)} e^{i / \hbar \int_{-\infty}^{\infty}(H(\nu(t))-H(\mu(t))) d t}\left\langle 0\left|\rho_{-\infty}\right| 0\right\rangle .
$$

Note that, as might have been expected, there is no non-trivial way that fine-grained histories of this sort will decohere.

\subsection{The decoherence functional as a trace over the Hilbert space $\mathcal{V}_{\text {cts }} \otimes \mathcal{V}_{\text {cts }}$}

The aim now is to write 3.15$)$ in the form

$$
d(\mu, \nu)=\operatorname{tr}_{\mathcal{V}_{\text {cts }} \otimes \mathcal{V}_{\text {cts }}}\left\{P_{|\exp \mu(\cdot)\rangle} \otimes P_{|\exp \nu(\cdot)\rangle} X\right\}
$$

where $P_{|\exp \mu(\cdot)\rangle}$ and $P_{|\exp \nu(\cdot)\rangle}$ are the bona fide projection operators constructed in section 2 .

It is helpful to construct $X$ in stages. Firstly, consider the following trace of (3.13) in the case that the dynamics is trivial (i.e., the Hamiltonian is zero)

$$
\operatorname{tr}_{L^{2}(\mathbb{R})}\left(\prod_{t \in \mathbb{R}} P_{\lambda(t)}\right)=e^{\int_{-\infty}^{\infty} \lambda^{*} d \lambda}
$$

where we have used the fact that $\lambda$ is square integrable, and hence vanishes at $\pm \infty$. We wish to write this as a trace over $\mathcal{V}_{\text {cts }}=\otimes_{t} L_{t}^{2}(\mathbb{R})$ i.e., we wish to find an operator $S_{\text {cts }}$ such that

$$
\operatorname{tr}_{L^{2}(\mathbb{R})}\left(\prod_{t \in \mathbb{R}} P_{\lambda(t)}\right)=\operatorname{tr}_{\mathcal{V}_{\text {cts }}}\left(S_{\text {cts }} P_{|\exp \lambda(\cdot)\rangle}\right)
$$

In order to understand how to construct $S_{\text {cts }}$, let us recall first what happens for a discrete, $n$-time history. As shown in [3], in this case we can write

$$
\operatorname{tr}_{L^{2}(\mathbb{R})}\left(A_{1} A_{2} \ldots A_{n}\right)=\operatorname{tr}_{\otimes^{n} L^{2}(\mathbb{R})}\left(A_{1} \otimes A_{2} \otimes \ldots \otimes A_{n} S_{n}\right)
$$

where $S_{n}$ is the operator on the $n$-fold tensor product space $\otimes^{n} L^{2}(\mathbb{R})$ that acts on the vector $v_{1} \otimes v_{2} \otimes \ldots \otimes v_{n} \in \otimes^{n} L^{2}(\mathbb{R})$ as

$$
S_{n}\left(v_{1} \otimes v_{2} \otimes \ldots \otimes v_{n}\right)=v_{2} \otimes v_{3} \otimes \ldots \otimes v_{n} \otimes v_{1} .
$$


For the purposes of the present paper it is important to note that $S_{n}$ is closely related to a discrete version of the derivative operator $\Delta_{n}$. If we take the time step $\delta t$ to have value one, we can write the action of $\Delta_{n}$ on a function symbolically as

$$
\Delta_{n} f\left(x_{r}\right)=f\left(x_{r+1}\right)-f\left(x_{r}\right)
$$

i.e., $\Delta_{n}$ corresponds to the matrix

$$
\Delta_{n}=\left(\begin{array}{lllll}
-1 & 1 & 0 & & \\
0 & -1 & 1 & & \\
& & & \ddots & \\
& & & & \ldots
\end{array}\right)
$$

Although $S_{n}$ acts on the $n$-fold tensor product and not on a direct sum of Hilbert spaces, one can nevertheless use the same notation to characterise its action as

$$
S_{n}\left(\begin{array}{l}
v_{1} \\
v_{2} \\
\vdots \\
\vdots \\
v_{n}
\end{array}\right)=\left(\begin{array}{l}
v_{2} \\
\vdots \\
\vdots \\
v_{n} \\
v_{1}
\end{array}\right)
$$

i.e.,

$$
S_{n}=\left(\begin{array}{cccc}
0 & 1 & 0 & \\
0 & 0 & 1 & \\
& & & \ddots
\end{array}\right)
$$

In other words, and returning to the continuous case, we might expect that $S_{\text {cts }}$ is roughly $1+\Delta$. Therefore, we might try to define $S_{\text {cts }}$ by

$$
S_{\text {cts }}|\exp \nu(\cdot)\rangle=|\exp (\nu(\cdot)+\dot{\nu}(\cdot))\rangle .
$$

or, perhaps better, define the operator $S_{\text {cts }}$ by its matrix elements:

$$
\left\langle\exp \mu(\cdot)\left|S_{\text {cts }}\right| \exp \nu(\cdot)\right\rangle \stackrel{\text { def }}{=} e^{\langle\mu, \nu\rangle+\langle\mu, \dot{\nu}\rangle}
$$

where

$$
e^{\langle\mu, \dot{\nu}\rangle} \stackrel{\text { def }}{=} e^{\int_{-\infty}^{\infty} \mu^{*} d \nu}
$$


in which the right hand side is a Stieltjes integral. Note that (3.26) defines $S_{\text {cts }}$ uniquely since the set of vectors of the form $|\exp \nu(\cdot)\rangle$, where $\nu$ is differentiable, is total.

The next step is to calculate the trace of the operator $S_{\operatorname{cts}} P_{|\exp \lambda(\cdot)\rangle}$ on the Hilbert space $\mathcal{V}_{\text {cts }}$. In the case of simple non-normalised coherent states $|\exp z\rangle$ on $L^{2}(\mathbb{R})$, the trace of any trace-class operator $T$ can be written as

$$
\operatorname{tr}(T)=\int d \kappa(z)\langle\exp z|T| \exp z\rangle
$$

where

$$
d \kappa(z)=\frac{1}{\pi} e^{-|z|^{2}} d(\Re z) d(\Im z)
$$

and where $\Re z$ and $\Im z$ denote the real and imaginary parts respectively of the complex number $z$.

The important thing for us is the analogue of this expression for the exponential Hilbert space. The key step here is the observation that the exponential Hilbert space $\exp L^{2}(\mathbb{R})$ is isomorphic to a Hilbert space of functionals on $L^{2}(\mathbb{R})$ whose inner product is defined via a certain Gaussian measure $\mathcal{D} \mu$. The details are standard [22] (albeit a little tricky in the functional analysis sense) and, for our purposes, it suffices to say that the generating functional for this measure has the property

$$
\int \mathcal{D} \mu[\nu] e^{\langle\nu, \lambda\rangle+\langle\rho, \nu\rangle}=e^{\langle\rho, \lambda\rangle} .
$$

It can be shown that the trace of a trace-class operator $T$ on the exponential Hilbert space can be evaluated using this measure as

$$
\operatorname{tr}(T)=\int \mathcal{D} \mu[\nu]\langle\exp \nu(\cdot)|T| \exp \nu(\cdot)\rangle
$$

which is the desired analogue of (3.28).

We may thus calculate $\operatorname{tr}_{\mathcal{V}_{\text {cts }}}\left(S_{\text {cts }} P_{|\exp \lambda(\cdot)\rangle}\right)$ as

$$
\begin{aligned}
& \operatorname{tr}_{\mathcal{V}_{\text {cts }}}\left(S_{\text {cts }} P_{|\exp \lambda(\cdot)\rangle}\right)=\int \mathcal{D} \mu[\nu]\left\langle\exp \nu(\cdot)\left|S_{\text {cts }} P_{|\exp \lambda(\cdot)\rangle}\right| \exp \nu(\cdot)\right\rangle \\
& =\int \mathcal{D} \mu[\nu]\left\langle\exp \nu(\cdot)\left|S_{\mathrm{cts}}\right| \exp \lambda(\cdot)\right\rangle e^{\langle\lambda, \nu-\lambda\rangle}
\end{aligned}
$$




$$
\begin{aligned}
& =\int \mathcal{D} \mu[\nu] e^{\langle\nu, \lambda+\dot{\lambda}\rangle+\langle\lambda, \nu-\lambda\rangle} \\
& =e^{\langle\lambda, \lambda+\dot{\lambda}\rangle-\langle\lambda, \lambda\rangle} \\
& =e^{\langle\lambda, \dot{\lambda}\rangle} \\
& =e^{\int_{-\infty}^{\infty} \lambda^{*} d \lambda}
\end{aligned}
$$

which is in agreement with (3.17), as desired.

The next step is to introduce dynamics, which we do by finding an operator $\mathcal{U}$ such that

$$
e^{\langle\lambda, \dot{\lambda}\rangle} e^{\frac{i}{\hbar} H[\lambda]}=\operatorname{tr}_{\mathcal{V}_{\text {cts }}}\left(S_{\text {cts }} \mathcal{U} P_{|\exp \lambda(\cdot)\rangle}\right)
$$

where $H[\lambda] \stackrel{\text { def }}{=} \int_{-\infty}^{\infty} H(\lambda(t)) d t$, and where we note that, from (3.13), the left hand side of (3.33) is $\langle 0|U(-\infty, \infty)| 0\rangle^{-1} \operatorname{tr}\left(\prod_{t \in \mathbb{R}} P_{\lambda(t)}(t)\right)$. The trace on the right hand side of (3.33) can be evaluated as

$$
\begin{aligned}
\operatorname{tr}_{\mathcal{V}_{\text {cts }}}( & S_{\text {cts }} \\
& \mathcal{U} P_{|\exp \lambda(\cdot)\rangle)} \\
& =\int \mathcal{D} \mu[\eta]\left\langle\exp \eta(\cdot)\left|S_{\text {cts }} \mathcal{U} P_{|\exp \lambda(\cdot)\rangle}\right| \exp \eta(\cdot)\right\rangle \\
& =\int \mathcal{D} \mu[\eta]\left\langle\exp \eta(\cdot)\left|P_{|\exp \lambda(\cdot)\rangle} S_{\text {cts }} \mathcal{U} P_{|\exp \lambda(\cdot)\rangle}\right| \exp \eta(\cdot)\right\rangle \\
& =\int \mathcal{D} \mu[\eta] e^{\langle\eta-\lambda, \lambda\rangle}\left\langle\exp \lambda(\cdot)\left|S_{\text {cts }} \mathcal{U}\right| \exp \lambda(\cdot)\right\rangle e^{\langle\lambda, \eta-\lambda\rangle} \\
& =\left\langle\exp \lambda(\cdot)\left|S_{\text {cts }} \mathcal{U}\right| \exp \lambda(\cdot)\right\rangle e^{-2\langle\lambda, \lambda\rangle} \int \mathcal{D} \mu[\eta] e^{\langle\eta, \lambda\rangle+\langle\lambda, \eta\rangle} \\
& =\left\langle\exp \lambda(\cdot)\left|S_{\text {cts }} \mathcal{U}\right| \exp \lambda(\cdot)\right\rangle e^{-\langle\lambda, \lambda\rangle} .
\end{aligned}
$$

Hence we are able to write the trace of the product of projection operators $\operatorname{tr}_{L^{2}(\mathbb{R})}\left(\prod_{t \in \mathbb{R}} P_{\lambda(t)}(t)\right)$ as $\langle 0|U(-\infty, \infty)| 0\rangle \operatorname{tr}_{\mathcal{V}_{\text {cts }}}\left(S_{\text {cts }} \mathcal{U} P_{|\exp \lambda(\cdot)\rangle}\right)$ using the operator $S_{\text {cts }} \mathcal{U}$ defined by

$$
\left\langle\exp \lambda(\cdot)\left|S_{\text {cts }} \mathcal{U}\right| \exp \lambda(\cdot)\right\rangle=e^{\langle\lambda, \lambda+\dot{\lambda}\rangle} e^{\frac{i}{\hbar} H[\lambda]}=\left\langle\exp \lambda(\cdot)\left|S_{\text {cts }}\right| \exp \lambda(\cdot)\right\rangle e^{\frac{i}{\hbar} H[\lambda]}
$$

While we shall not need it explicitly in what follows, it is not difficult to show that the matrix elements of $\mathcal{U}$ are given by

$$
\begin{aligned}
\langle\exp \eta(\cdot)|\mathcal{U}| \exp \nu(\cdot)\rangle= & e^{\left(\left\langle\left(1+\frac{\partial}{\partial t}\right)^{-1 \dagger} \eta, \frac{\delta}{\delta \lambda}\right\rangle+\left\langle\frac{\delta}{\delta \lambda}, \mu\right\rangle\right)} \\
& \times\left. e^{\langle\lambda, \lambda+\dot{\lambda}\rangle} e^{\frac{i}{\hbar} H[\lambda]}\right|_{\lambda=\bar{\lambda}=0}
\end{aligned}
$$


where we have used the fact that, for coherent states, the off-diagonal matrix elements of any operator $A$ are given in terms of the diagonal ones by

$$
\langle\exp \eta(\cdot)|A| \exp \nu(\cdot)\rangle=\left.e^{\left(\left\langle\eta, \frac{\delta}{\delta \lambda}\right\rangle+\left\langle\frac{\delta}{\delta \lambda}, \nu\right\rangle\right)}\langle\exp \lambda(\cdot)|A| \exp \lambda(\cdot)\rangle\right|_{\lambda=\bar{\lambda}=0}
$$

We are now in a position to express the decoherence functional in terms of a trace over $\mathcal{V}_{\text {cts }} \otimes \mathcal{V}_{\text {cts. }}$. We recall that, from (3.15),

$$
\begin{aligned}
d(\mu, \nu) & =\operatorname{tr}_{L^{2}(\mathbb{R})}\left(\left(\prod_{t \in \mathbb{R}} P_{\mu(t)}\right)^{\dagger} \rho_{-\infty}\left(\prod_{t \in \mathbb{R}} P_{\nu(t)}\right)\right) \\
& =e^{\int_{-\infty}^{\infty}\left(\nu^{*} d \nu-\mu^{*} d \mu\right)} e^{i / \hbar \int_{-\infty}^{\infty}(H(\nu(t))-H(\mu(t))) d t}\left\langle 0\left|\rho_{-\infty}\right| 0\right\rangle .
\end{aligned}
$$

Thus, using the operator $S_{\text {cts }} \mathcal{U}$ defined by (3.35), we get

$$
\begin{aligned}
d(\mu, \nu) & =\operatorname{tr}_{\mathcal{V}_{\text {cts }}}\left(S_{\text {cts }} \mathcal{U} P_{|\exp \mu(\cdot)\rangle}\right)^{*} \operatorname{tr}_{\mathcal{V}_{\text {cts }}}\left(S_{\text {cts }} \mathcal{U} P_{|\exp \nu(\cdot)\rangle}\right)\left\langle 0\left|\rho_{-\infty}\right| 0\right\rangle \\
& =\operatorname{tr}_{\mathcal{V}_{\text {cts }}}\left(\left(S_{\text {cts }} \mathcal{U}\right)^{\dagger} P_{|\exp \mu(\cdot)\rangle}\right) \operatorname{tr}_{\mathcal{V}_{\text {cts }}}\left(S_{\text {cts }} \mathcal{U} P_{|\exp \nu(\cdot)\rangle}\right)\left\langle 0\left|\rho_{-\infty}\right| 0\right\rangle \\
& =\operatorname{tr}_{\mathcal{V}_{\text {cts }} \otimes \mathcal{V}_{\text {cts }}}\left(P_{|\exp \mu(\cdot)\rangle} \otimes P_{|\exp \nu(\cdot)\rangle} X\right)
\end{aligned}
$$

where

$$
X \stackrel{\text { def }}{=}\left\langle 0\left|\rho_{-\infty}\right| 0\right\rangle\left(S_{\text {cts }} \mathcal{U}\right)^{\dagger} \otimes\left(S_{\text {cts }} \mathcal{U}\right)
$$

\section{Conclusion}

We have shown how the history group of a continuous-time history theory leads to a certain natural class of projection operators in a continuous tensor-product space. We have also shown how to construct class operators as continuous products of projection operators, and we demonstrated how the decoherence function of a pair of class operators can be re-expressed in the tensor product space using the projection operators that represent the history propositions. The calculations of decoherence functionals performed above are restricted to histories associated with projectors onto coherent states: an important task for the future therefore is to extend these calculations to include projectors that correspond to temporal averages of physical quantities. 
The history group plays a central role in these constructions by giving us the Hilbert space $\mathcal{V}_{\text {cts }}$ whose projectors form the space of history propositions, just as the canonical group gives the Hilbert space of standard, single-time quantum mechanics. The fact that the history group of quantum mechanics is the same as the canonical group of a $1+1$-dimensional field theory raises several important issues. The first is the existence of many unitarily inequivalent representations: a feature whose relevance for the history theory remains to be seen. Another is the intriguing possibility that arises in the case of a spin system. The single-time canonical commutation relations are

$$
\left[S^{i}, S^{j}\right]=i \epsilon^{i j k} S^{k}, \quad i, j, k=1,2,3
$$

which suggests that the relevant history group has the Lie algebra

$$
\left[S_{t}^{i}, S_{t^{\prime}}^{j}\right]=i \epsilon^{i j k} S_{t^{\prime}}^{k} \delta\left(t-t^{\prime}\right), \quad i, j, k=1,2,3 .
$$

However, unlike (4.1), the algebra (4.2) can have a non-trivial central extension, which raises the possibility that the history algebra for a spin system might instead be

$$
\left[S_{t}^{i}, S_{t^{\prime}}^{j}\right]=i \epsilon^{i j k} S_{t^{\prime}}^{k} \delta\left(t-t^{\prime}\right)+i \kappa \delta^{i j} \partial_{t} \delta\left(t-t^{\prime}\right)
$$

where $\kappa$ is a constant. The representation theories of (4.2) and (4.3) are, of course, very different. The investigation of the physical implications of using (4.3) rather than (4.2) will be left to future work.

There is another feature of the history framework that does not occur in the standard single-time treatment, and which deserves further study: the fact that there are 'temporally entangled' states in the history theory. While this feature appears in the case of continuous histories studied in this paper, it also occurs in a theory with only discrete-time histories, and is perhaps simpler to describe there.

Consider the case of standard quantum mechanics, and let us consider only two-time histories. If the Hilbert space of the canonical theory is $\mathcal{V}$, then the Hilbert space upon which the history propositions are projection operators is $\mathcal{V} \otimes \mathcal{V}$. As well as the homogeneous histories, (projectors onto vectors of the form $|u\rangle \otimes|v\rangle$ ) and the inhomogeneous histories made from them by the appropriate logical operations (e.g. disjoint union $\oplus$ ), the space 
of projectors on $\mathcal{V} \otimes \mathcal{V}$ also includes certain 'exotic histories', for example the projector onto the suitably normalised vector

$$
\left(\left|u_{1}\right\rangle \otimes\left|v_{1}\right\rangle+\left|u_{2}\right\rangle \otimes\left|v_{2}\right\rangle\right)
$$

In the case of the single-time quantum theory of a composite system, this is the sort of entangled state that leads to many of the peculiar features of quantum mechanics. In the case of the history framework, we see that there is the possibility of a quite new type of quantum entanglement.

\section{Acknowledgements}

We would like to thank Jim Hartle for many helpful discussions during early stages of the work. We gratefully acknowledge support from the SERC, from Trinity College, Cambridge (CJI), and the Leverhulme and Newton Trusts (NL). The work described in this paper was started at the Isaac Newton Institute, and we are both grateful to the staff there for providing a stimulating working environment.

\section{References}

[1] C.J. Isham. Quantum logic and the histories approach to quantum theory. J. Math. Phys. , 23:2157-2185, 1994.

[2] C.J. Isham and N. Linden. Quantum temporal logic and decoherence functionals in the histories approach to generalised quantum theory. $J$. Math. Phys. 35:5452-5476, 1994.

[3] C.J. Isham, N. Linden and S. Schreckenberg. The classification of decoherence functionals: an analogue of Gleason's theorem. J. Math. Phys. 35:6360-6370, 1994.

[4] R.B. Griffiths. Consistent histories and the interpretation of quantum mechanics. J. Stat. Phys., 36:219-272, 1984.

[5] R. Omnès. Logical reformulation of quantum mechanics. I. Foundations. J. Stat. Phys., 53:893-932, 1988. 
[6] R. Omnès. Logical reformulation of quantum mechanics. II. Interferences and the Einstein-Podolsky-Rosen experiment. J. Stat. Phys., 53:933955, 1988.

[7] R. Omnès. Logical reformulation of quantum mechanics. III. Classical limit and irreversibility. J. Stat. Phys., 53:957-975, 1988.

[8] R. Omnès. Logical reformulation of quantum mechanics. III. Projectors in semiclassical physics. J. Stat. Phys., 57:357-382, 1989.

[9] R. Omnès. From Hilbert space to common sense: A synthesis of recent progress in the interpretation of quantum mechanics. Ann. Phys. (NY), 201:354-447, 1990 .

[10] R. Omnès. Consistent interpretations of quantum mechanics. Rev. Mod. Phys., 64:339-382, 1992.

[11] M. Gell-Mann and J. Hartle. Quantum mechanics in the light of quantum cosmology. In S. Kobayashi, H. Ezawa, Y. Murayama, and S. Nomura, editors, Proceedings of the Third International Symposium on the Foundations of Quantum Mechanics in the Light of New Technology, pages 321-343. Physical Society of Japan, Tokyo, 1990.

[12] M. Gell-Mann and J. Hartle. Quantum mechanics in the light of quantum cosmology. In W. Zurek, editor, Complexity, Entropy and the Physics of Information, SFI Studies in the Science of Complexity, Vol. VIII, pages 425-458. Addison-Wesley, Reading, 1990.

[13] M. Gell-Mann and J. Hartle. Alternative decohering histories in quantum mechanics. In K.K. Phua and Y. Yamaguchi, editors, Proceedings of the 25th International Conference on High Energy Physics, Singapore, August, 2-8, 1990, Singapore, 1990. World Scientific.

[14] J. Hartle. The quantum mechanics of cosmology. In S. Coleman, J. Hartle, T. Piran, and S. Weinberg, editors, Quantum Cosmology and Baby Universes. World Scientific, Singapore, 1991.

[15] J. Hartle. Spacetime grainings in nonrelativistic quantum mechanics. Phys. Rev., D44:3173-3195, 1991. 
[16] M. Gell-Mann and J. Hartle. Classical equations for quantum systems. 1992. UCSB preprint UCSBTH-91-15.

[17] J. Hartle. Spacetime quantum mechanics and the quantum mechanics of spacetime. In Proceedings on the 1992 Les Houches School, Gravitation and Quantisation. 1993.

[18] D.J. Foulis, R.J. Greechie, and G.T. Rüttimann. Filters and supports in orthoalgebras. Int. J. Theor. Phys., 31:789-807, 1992.

[19] H Araki and E.J. Woods. Complete Boolean algebras of type 1 factors. Publ. Res. Inst. Maths. Sci. (Kyoto) Ser A, 2:157-242, 1966.

[20] J.R. Klauder. Exponential Hilbert space: Fock space revisited. J. Math. Phys., 2:609-630, 1969.

[21] R.F. Streater. Current commutation relations, continuous tensor products and infinitely divisible group representations. Rend. Sci. Int. Fisica E. Fermi, XI:247-263, 1969.

[22] A. Guichardet. Symmetric Hilbert spaces and related topics, Lecture notes in mathematics, Vol. 261, Springer-Verlag, Berlin, 1972.

[23] J. Erven and B.-J. Falkowski. Low order cohomology and applications, Lecture notes in mathematics, Vol. 877, Springer-Verlag, Berlin, 1981.

[24] J.R. Klauder and B-S. Skagerstam. Coherent States. World Scientific, Singapore, 1985. 